\title{
A LOUD-SPEAKER STETHOSCOPE FOR CLINICAL TEACHING
}

\author{
BY \\ A. F. PHILLIPS* \\ From the Department of Physiology, University of Edinburgh \\ Received September 18, 1948
}

An equipment was required that would enable a number of students to listen simultaneously in auscultation of a patient's chest. The method adopted is to pick up the sounds by means of a microphone held on the chest, amplify them electrically, and reproduce them at a loud-speaker. The problem differs from that of phonocardiography in two main respects: first, the subjective effect on the listener must be as similar as possible to auscultation with a stethoscope, which means that certain components of the sounds, too small to affect the phonocardiograph record, must be faithfully reproduced, and at the same time frequencies that are not well picked up by a stethoscope must be correspondingly attenuated by the electrical system; and secondly, oscillation ("howling ") is liable to occur due to the microphone picking up the air-borne sound from the loud-speaker.

There is also a fundamental difficulty. The relative sensitivity of the human ear to different frequencies depends on the amplitude of the sound, so that if, by one setting of the amplifier, a student at a certain distance from the loud-speaker hears exactly what he would hear with a particular stethoscope, another at a different distance will not get the same subjective effect. If a high standard of fidelity is demanded, this fact probably limits the method to fairly small groups.

Again, students at a little distance from the patient are at a disadvantage with regard to discrimination, conscious or subconscious, against sounds due to accidental movements of the chestpiece on the skin, and to discrimination against breath sounds when listening to heart sounds and murmurs, because they cannot see small movements. Thus it might be that the best results would be obtained by a compromise that sacrificed fidelity slightly in order to reduce the relative amplitude of such sounds.

Finally there are technical difficulties due to the very low frequencies of the important sounds, which go below the range of all but the best commercial acoustic equipment, and due to the great range of amplitude from the large very low-frequency components of the first heart-sound to the smallest audible murmur.

In the equipment described in this paper, the main difficulties have been overcome, and satisfactory reproduction is believed to have been achieved for at least twenty students at a time. No actual teaching has been done with the equipment at the time of writing. The total cost, apart from time, was about $£ 70$.

\section{Discussion of the Problem in the Light OF Previous Work}

The equipment is required to pick up from the chest normal and pathological cardiac and respiratory sounds, and to reproduce them by a loudspeaker to give as nearly as possible the same effect as auscultation with a stethoscope. Extraneous sounds, such as those due to friction on the skin and air-borne sounds of all kinds, are not required and should be reproduced as little as possible consistent with the main requirement. For the design of the equipment, therefore, information is required on the frequency response of stethoscopes and on the range of frequency covered by the wanted sounds. The equipment should be designed so that the frequency response is the same as that of a stethoscope over this wanted range of frequency, and outside this range the less the response the better.

Cabot and Dodge (1925) studied the frequency distribution of heart and breath sounds, mainly pathological, over the range 30 to $2600 \mathrm{c} / \mathrm{s}$, by a method that is directly applicable to the present problem. They switched different filters in turn into their amplifier and listened for any change in the quality of the sounds as reproduced by it. They

* Vans Dunlop Scholar in Physiology, University of Edinburgh. 
found that all components of heart sounds and murmurs were below $1000 \mathrm{c} / \mathrm{s}$, and most were below $660 \mathrm{c} / \mathrm{s}^{\circ}$ The lower limit was in some cases $120 \mathrm{c} / \mathrm{s}$, but in others there were components down to the lower limit of their apparatus. Breath sounds were almost entirely within the range 120 to $1000 \mathrm{c} / \mathrm{s}$. At the date of their work the electrical equipment can hardly have been good enough to give a high standard of fidelity. Brooker (1946), who describes the equipment used in making gramophone recordings for Stokes (1946), found that although most of the energy in the sounds was below $1500 \mathrm{c} / \mathrm{s}$, an amplifier that cut off above $2000 \mathrm{c} / \mathrm{s}$ did not give perfect reproduction, and he used one which went up to $4500 \mathrm{c} / \mathrm{s}$ in spite of the accompanying disadvantage of increased background noise. The lower limit of his amplifier was $30 \mathrm{c} / \mathrm{s}$, but he believed that lower frequencies were present and influenced the total subjective effect.

Mannheimer (1940) has analysed heart sounds and murmurs in normal children and children with congenital heart disease. He used amplifiers incorporating combinations of good filters with measured characteristics, covering a number of frequency bands, the lowest being 0 to $100 \mathrm{c} / \mathrm{s}$ and the highest 500 to $1000 \mathrm{c} / \mathrm{s}$. In different cases he found components both of normal sounds and of murmurs in all his frequency bands. His apparatus (which was primarily for phonocardiography) cut off all components above $1000 \mathrm{c} / \mathrm{s}$, and this he found advantageous in reducing the effect of air-borne disturbances.

The absolute measurement of the frequency response of a stethoscope is difficult. In the conditions in which it is used, the frequency response depends not only on the design of the instrument itself, but also on the degree of acoustic mismatching both where the sound is transferred from the patient's body to the chest-piece and where it is transferred from the ear-pieces to the physician's ears, and unless special precautions are taken the degree of mis-matching at one or both places will be different under the conditions of the experiment from that under practical working conditions. It is of course also necessary to know and correct for the frequency response of the source of sound (e.g. a loud-speaker), and of the microphone and other apparatus used for measuring the amplitude transmitted by the stethoscope. No account has been found of a measurement of this kind in which a full technical description of the apparatus has been given, and it seems likely that authors have failed to realize the importance of reproducing exactly the acoustical mis-match which occurs in practice. A technique in which these matters are fully taken account of, is described in a Medical Research Council Special
Report (1947), and was used for measuring the performance of hearing aids. Some such technique could be applied to measurement of the frequency response of a stethoscope, but as far as I know this has not been done.

Rappaport and Sprague (1941) have attempted to measure the frequency response of stethoscopes and give a theoretical response curve for an amplifying stethoscope which should reproduce the effect of " a theoretically average acoustical stethoscope," but it does not seem that their technique was adequate for the reasons just discussed. Their curve falls off steadily from $200 \mathrm{c} / \mathrm{s}$ downwards, to $-20 \mathrm{db}$ at $30 \mathrm{c} / \mathrm{s}$.

Comparison of the effects of different stethoscope chest-pieces is not so difficult. By putting a source of sound in the heart of a cadaver and applying the different chest-pieces to the chest, Johnstone and Kline (1940) closely simulated the conditions of clinical use as far as the acoustical mis-match at that end was concerned. They compared different stethoscopes over the range 20 to $800 \mathrm{c} / \mathrm{s}$, and from their curves it appears that the main effect of a diaphragm, compared with an open bell, is to attenuate the lower frequencies and so increase the relative high-frequency response.

Many whole loud-speaker equipments for teaching are mentioned in papers from 1920 onwards, but usually without precise information about frequency response. Gamble and Replogle (1924) describe the apparatus used by Cabot (1923), and Gamble (1924) describes improvements to the apparatus and the results of experience after a few months' use. Their trials with loud-speaker reproduction were unsatisfactory, and they used a telephone type of output device to which an ordinary stethoscope was applied. They found that a filter cutting off sounds above $660 \mathrm{c} / \mathrm{s}$ gave the best results for routine use, and that additional filters were useful for accentuating particular sounds and murmurs, in particular one passing frequencies below $140 \mathrm{c} / \mathrm{s}$ and one passing frequencies above $130 \mathrm{c} / \mathrm{s}$. Rappaport and Sprague (1941) discuss the reasons for poor results with loudspeaker equipments, and emphasize the importance of adequate loud-speaker power-handling capacity and freedom from resonances, as well as correct frequency response of the whole equipment. The advantages of loud-speaker reproduction for teaching purposes are discussed by Henriques (1937).

As regards choice of microphone, since the introduction of the piezo-electric crystal microphone this has been unanimously accepted as the best type for the purpose (e.g. by Sacks and Marquis (1935) and Rentschler (1936), etc.). My own experiments with a few other types which happened to be available agree with this. 
Characteristics Required for the EquipMent

The simplest method of getting a controlled frequency response is to use a microphone and a loudspeaker that both have a response approximately independent of frequency over the range concerned, and to incorporate suitable filters in the amplifier. The range to be covered is from about $30 \mathrm{c} / \mathrm{s}$ up to at least $1000 \mathrm{c} / \mathrm{s}$, and probably up to $4000 \mathrm{c} / \mathrm{s}$.

A piezo-electric crystal microphone covers the frequency range satisfactorily; but because of its high impedance it is necessary to keep the capacity of the lead to the first stage of the amplifier low (in the region of 0.0001 microfarad) in order to maintain the response at the upper end of the frequency range. The amplifier input impedance must be high; suitable input circuits are given in Radio Designer's Handbook, chapter XI. It is desirable to separate the amplifier from the microphone so that the latter can be mounted in a small light unit, to be placed directly on the chest. High sensitivity to vibrations from the chest-wall, and low sensitivity to air-borne sound and to friction on the case, are required.

The loud-speaker must have a response maintained to the lowest audible frequencies, and portability is an advantage. It must be free from resonances in the lower audio-frequency range, and must also have a power-handling capacity much greater than the mean power to be broadcast, because the very large amplitude low-frequency components in the heart-sounds, which are almost inaudible themselves because of their low frequency, cause chattering or booming in a small loud-speaker.

The amplifier must have a response maintained up to about $4000 \mathrm{c} / \mathrm{s}$, and down to the lowest audible frequencies, though some falling off below $200 \mathrm{c} / \mathrm{s}$ is probably required to simulate a stethoscope frequency response. Adjustable filters are required to reduce the low-frequency response and simulate a diaphragm stethoscope, and to reduce the high-frequency response and discriminate against extraneous sounds, which tend to have higher frequencies than the wanted sounds. Further adjustment of the frequency response is desirable so that an attempt may be made to emphasize a particular sound or murmur. The filter and volume controls must be early enough in the amplifier circuit to avoid "limiting" at any stage, but as late as possible, consistent with this, to give quietness in operation. These controls should be within reach of the physician who holds the chestpiece. The distortion-free output of the amplifier need not be as great as the power-handling capacity of the loud-speaker, because the largest amplitudes are at frequencies that are very little heard as sound and do not need to be faithfully reproduced; indeed, a certain degree of relative reduction of the largest amplitudes is probably desirable.

Finally, simplicity of operation is important in an equipment to be used for teaching.

\section{Description of the Apparatus}

Apparatus has been assembled that fulfils very nearly the requirements set out in the previous section. The block diagram (Fig. 1) indicates the arrangement of the different units. A small microphone is used like a stethoscope chest-piece, and its output is carried by 6 feet of rubber-protected screened wire to the pre-amplifier. This is housed in a metal box $6 \times 6 \times 2 \frac{1}{4}$ inches, which also contains the volume control and the tone-control filters. This box lies on the bed or a locker, and the three controls, treble, bass, and volume, are operated by rotating knobs. Thus all the control can be done by the physician who handles the microphone. The output from the pre-amplifier and the power supplies to it are carried by a multi-core cable 11 feet long, connecting it with a larger box $17 \times 10 \times 9$ inches, which contains the main amplifier and all the power supply. (The whole apparatus is supplied from A.C. mains.) The output is led to the loud-speaker by 20 feet of twin flex, with an optional extension of another 35 feet. The loud-speaker cabinet is of wood, and measures $31 \times 18 \times 18$ inches.

Microphone. The microphone at present in use consists of a small piezo-electric crystal unit mounted at the apex of a hollow cone turned out of a cylin-

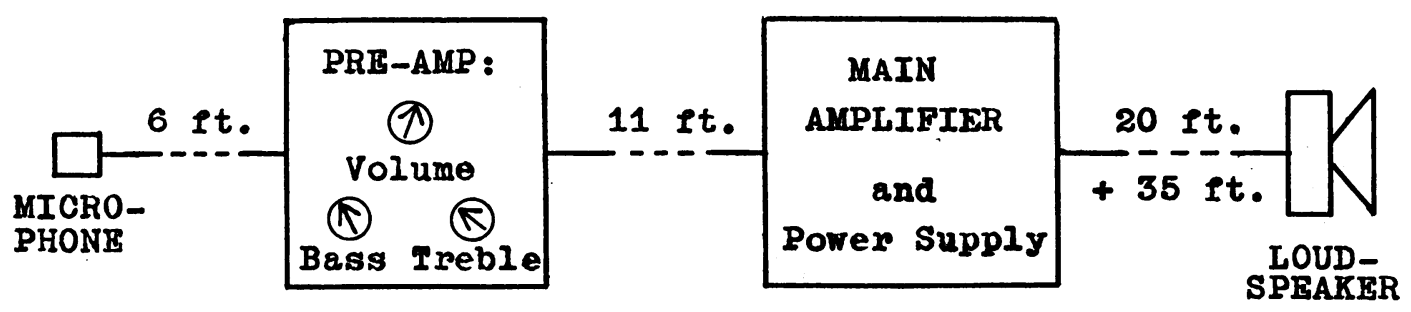

FIG. 1.-Block diagram of the complete loud-speaker stethoscope. 
drical block of brass. This is chromium-plated, and is held in the hand in direct contact with the chest. The diameter of the orifice is 1 inch; the total weight is $5 \mathrm{oz}$. Noises due to friction are no worse than with an ordinary stethoscope. Provided the microphone is in contact with the skin all round its rim, there is little tendency to " howl," and in most cases " howling " is not the factor that limits the maximum useful amplification. The frequency response of this type of microphone (without moving parts other than the crystal itself) can be made practically uniform, but here it is being used with a lower amplifier input impedance than is recommended, which probably reduces the response at the lower frequencies.

It is intended to try other types of crystal microphone, as further improvement may still be possible.

Amplifier. A high-quality audio-frequency amplifier* was modified to suit the special requirements. It consists of two triode stages as pre-amplifier giving a voltage gain $\times 850$, followed by a filter circuit with treble and bass controls and middle-frequency attenuation $\div 10(20 \mathrm{db})$, a potentiometer volume control, an amplifier and phase-splitter stage, and a push-pull power output stage. The amplifier gives an output of 12 watts for an input to the first stage of 20 millivolts r.m.s. The input impedance (first grid leak) is 1.5 megohm.

* The amplifier “QA12/P," supplied by The Acoustical Manufacturing Co. Ltd., Huntingdon.
The principal modification required was in the filter circuit, which is shown in its final form in Fig. 2. It is a resistance-capacity network with separate high-frequency and low-frequency controls, which, in the modified circuit, are not entirely independent in their effects. The frequency response curves for the middle and extreme settings of the two controls are shown in Fig. 3 and 4.

The filter controls and the volume control are silent in operation. No trouble was encountered from valve noise or microphony. In the cable connecting the pre-amplifier to the main amplifier there was considerable pick-up of hum from H.T. and L.T. supplies, which was overcome by additional smoothing in the case of the former, but for the L.T. a separate transformer was necessary, as spikes at $200 \mathrm{c} / \mathrm{s}$ recurrence were generated in the main transformer and were picked up by the signal lead from the heater leads. With these alterations, the hum from the equipment itself is barely audible, and the measured level in an electrical laboratory was $80 \mathrm{mv}$. r.m.s. across the $15-\mathrm{ohm}$ output, part of this being due to pick-up from other sources.

Loud-speaker. The loud-speaker is a $15-\mathrm{ohm}$, 15-watt, 12 inch unit in a special cabinet mounting.* This type of mounting is probably the best to combine robustness and portability with a good low-

* "Labyrinth Loud-speaker, Type SL.15," supplied by The Acoustical Manufacturing Co. Ltd., Huntingdon.

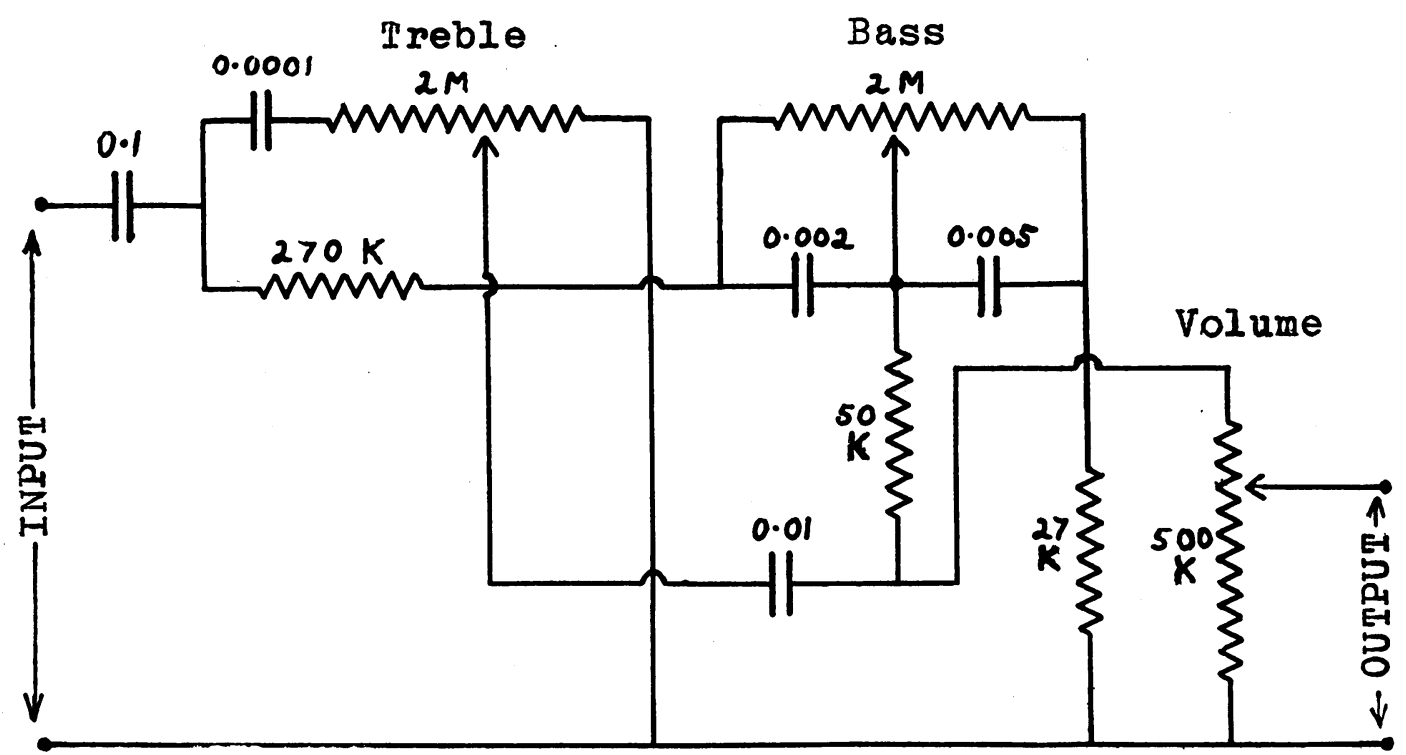

Fig. 2.-Full diagram of the filter circuit showing treble, bass, and volume controls. Resistances in thousands of ohms (K) and megohms (M). Capacities in fractions of a microfarad. Input: direct from anode of second triode amplifier stage, anode load $100 \mathrm{~K}$. Output: direct to grid of subsequent stage. 


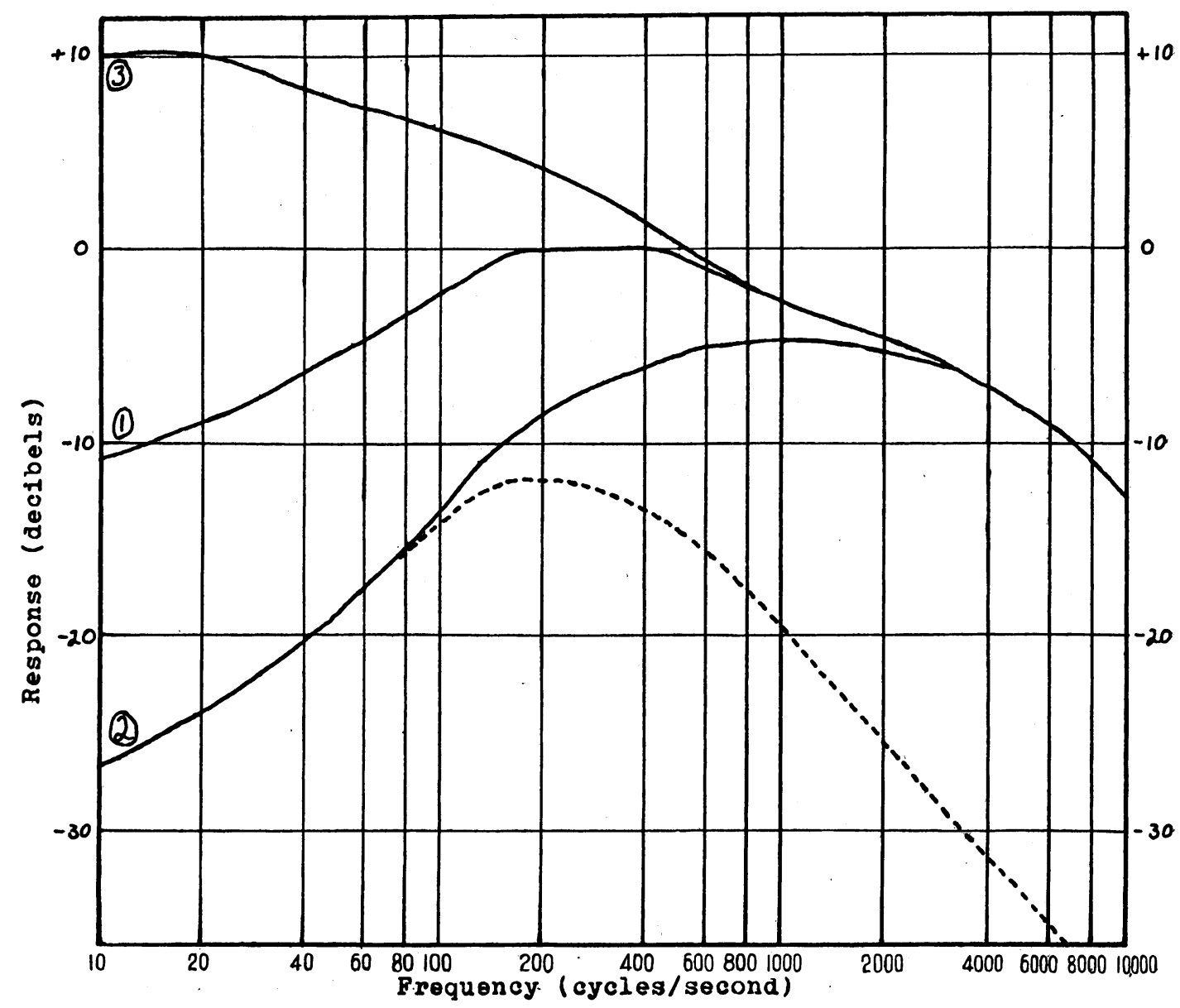

Fig. 3.-Frequency-response curves of amplifier to show the effect of the bass control.

Full curves: treble control central.

(1) Bass control also central.

(2) Bass control set at minimum.

(3) Bass control set at maximum.

Broken curve: both controls set at minimum.

frequency response. The published performance curve indicates a response that is practically independent of frequency down to $35 \mathrm{c} / \mathrm{s}$, with no significant resonances.

There is a slight tendency to boom at the first heart-sound, and this is in most cases the factor that limits the maximum volume consistent with good reproduction.

Connection to the loud-speaker is made by means of 20 feet of twin flex, with a jack which is plugged into the main amplifier box. An extension of 35 feet of twin flex is available, with a jack socket at one end and a plug at the other. Each socket is arranged to leave a $15-\mathrm{ohm}$ load across the output when the corresponding plug is removed, so that there is no danger of leaving the output transformer unloaded.

\section{RESUltS}

The equipment gives a very close approach to the effect of auscultation with a stethoscope, in a quiet room large enough for twenty people. For auscultation over the præcordium the apparent loudness cannot usefully be increased much above that heard with a stethoscope because distortion of the sounds begins to occur. A variety of pathological murmurs have been listened to, and these as well as the normal sounds are satisfactorily heard. On the whole, very 


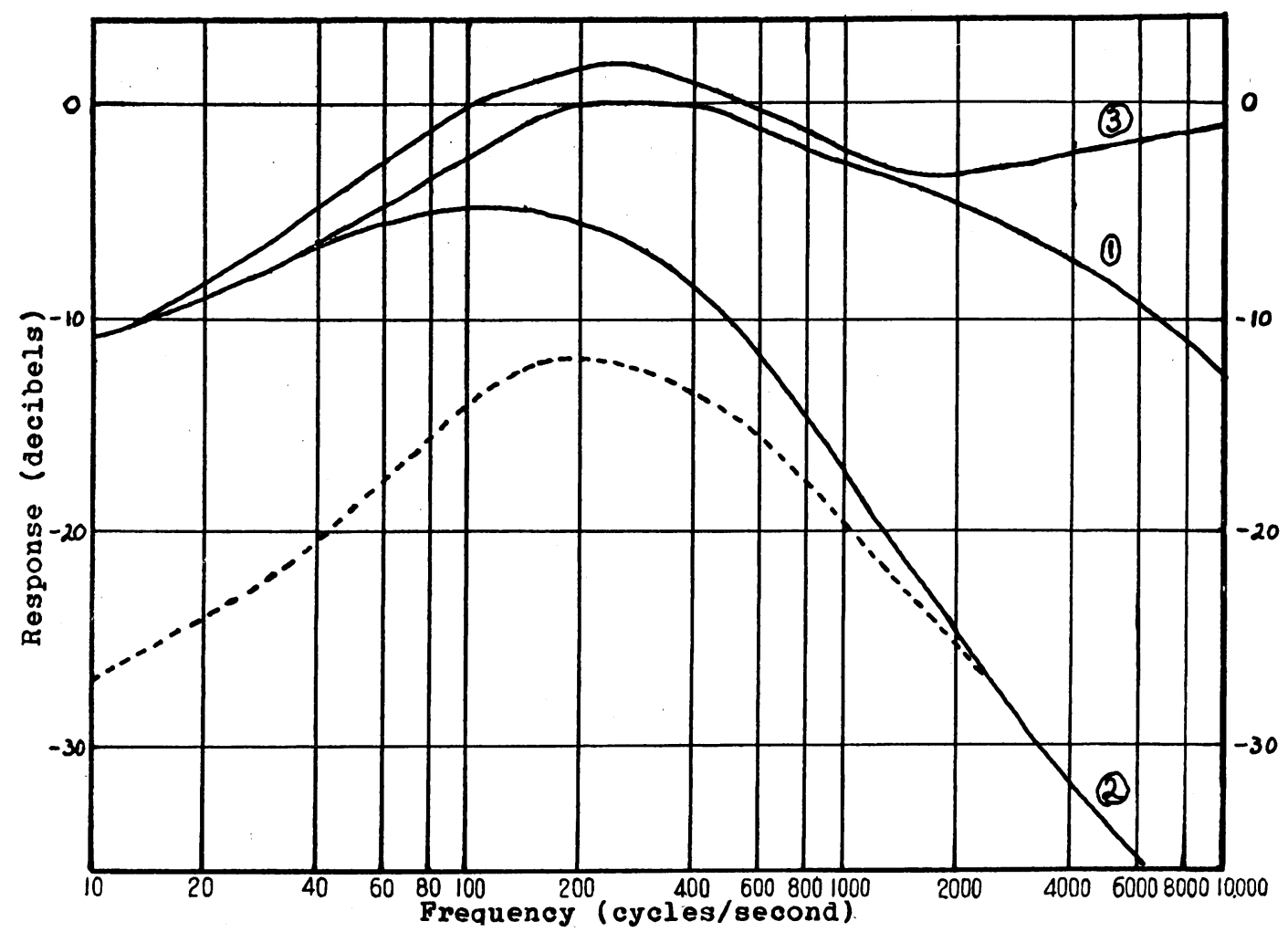

Fig. 4.-Frequency-response curves of amplifier to show the effect of the treble control.

Full curves: bass control central.

(1) Treble control also central.

(2) Treble control set at minimum.

(3) Treble control set at maximum.

Broken curve: both controls set at minimum.

faint murmurs are not more easily picked up with the loud-speaker than with a stethoscope, but simultaneous auscultation will allow each student to listen for a much longer time than he would otherwise be able to do, and so increase his chances of picking up a difficult murmur.

For auscultation of breath sounds the volume can be considerably increased without distortion, and both normal and pathological sounds are picked up much more easily than with a stethoscope. The volume can then of course be reduced to simulate a stethoscope more exactly.

\section{SUMMARY}

The problems of electrical amplification of heart sounds and of group auscultation are discussed both theoretically and with reference to previous work.
A portable equipment is described which is believed to be suitable for teaching groups of at least twenty students. This consists of a crystal microphone, an electrical amplifier, and a loudspeaker, which have been arranged to give an effect that simulates very closely auscultation with a stethoscope.

The amplification, and the high- and low-frequency response of the amplifier, can be easily and silently controlled during auscultation. The relevant frequency response curves are given.

This work was done during the tenure of a Vans Dunlop Scholarship in Physiology, at the University of Edinburgh.

The author wishes to thank Prof. A. E. Ritchie for the use of his laboratory and electrical apparatus and for his help at all stages of the work; and Dr. R. W. D. Turner for providing facilities to test the equipment on patients at the Western General Hospital, Edinburgh. 


\section{REFERENCES}

Brooker, V. M. (1946). Med. J. Aust., 2, 595.

Cabot, R. C. (1923). J. Amer. med. Ass., 81, 298. , and Dodge, H. F. (1925). Ibid., 84, 1793.

Gamble, C. J. (1924). Ibid., 83, 1230. and Replogle, D. E. (1924). Ibid., 82, 387.

Henriques, C. V. (1937). Lancet, 1937.1, 686.

Johnston, F. D., and Kline, E. M. (1940). Arch. intern. Med., 65, 328.

Mannheimer, E. (1940). Acta Paediatr., 28, supp. 2.
Medical Research Council, Special Report No. 261. (1947). London, H.M. Stationery Office.

Radio Designer's Handbook. 1st. British edit., p. 78. London, Iliffe \& Sons.

Rappaport, M. B., and Sprague, H. B. (1941). Amer. Heart J., 21, 257.

Rentschler, L. B. (1936). Science, 83, 627.

Sacks, H. A., and Marquís, H. (1935). Proc. Soc. exp. Biol. N.Y., 32, 773.

Stokes, E. H. (1946). Med. J. Aust., 1946.2, 595. 